\title{
Identification of PEM Fuel Cells based on Support Vector Regression and Orthonormal Bases
}

\author{
Diego Feroldi and Juan C. Gómez \\ CIFASIS-CONICET \\ FCEIA-Universidad Nacional de Rosario \\ Rosario, Argentina \\ fferoldi, gomez\}@ cifasis-conicet.gov.ar
}

\author{
Vicente Roda \\ Institut de Robótica i Informática Industrial \\ CSIC-UPC, Parc Tecnológic de Barcelona \\ Barcelona, Spain \\ vroda@iri.upc.edu
}

\begin{abstract}
Polymer Electrolyte Membrane Fuel Cells (PEMFC) are efficient devices that convert the chemical energy of the reactants in electricity. In this type of fuel cells, the performance of the air supply system is fundamental to improve their efficiency. An accurate mathematical model representing the air filling dynamics for a wide range of operating points is then necessary for control design and analysis. In this paper, a new Wiener model identification method based on Support Vector (SV) Regression and orthonormal bases is introduced and used to estimate a nonlinear dynamical model for the air supply system of a laboratory PEMFC from experimental data. The method is experimentally validated using a PEMFC system based on a ZBT ${ }^{\mathrm{R}}$ 8-cell stack with Nafion $115^{\mathrm{R}}$ membrane electrode assemblies.
\end{abstract}

\section{INTRODUCTION}

Polymer Electrolyte Membrane Fuel Cells (PEMFC) are one of the most advanced fuel cell systems, which allow an efficient transformation of the chemical energy stored in hydrogen into electric power. The efficiency of the whole system is strongly influenced by the air supply subsystem due to the compressor power consumption. One of the most important challenges in PEMFC control is then to guarantee that sufficient amount of oxygen is fed to the cathode with the objective of optimizing the net output power. Control of the air supply results critical because the oxygen reacts very fast as current is drawn, whereas the oxygen supply is limited by the dynamics of the inlet manifold and the air compressor [1].

Mathematical models can be used to describe the fundamental phenomena that take place in the system, to predict its behavior under different operating conditions, and to design and optimize the control of the system. Nevertheless, in spite of having available many PEMFC models in stationary state, few dynamic models have been developed. Dynamic models for PEMFC that are suitable for control studies have been introduced in [2] and [3]. These models capture the transient behavior of the air compressor, the gasses filling dynamics, and the effect of the membrane humidity. These variables affect the cell voltage and, therefore, the efficiency and the output power of the system. However, the models in [2] and [3] are difficult to parameterized and too complex to be used in model-based control strategies. In fact, most of the control schemes for PEMFC available in the literature (including the ones proposed in [2] and [3]) are based on linearized models of the fuel cells, and therefore they are of limited utility.

In recent years, some advanced computational techniques have been exploited to optimize the parameters of fuel cell models. In [4] the parameters of the fuel cell model are determined and optimized through a hybrid genetic algorithm (HGA) by using stack output-voltage and current, and anode and cathode pressure as input-output data. In [5] a Particle Swarm Optimization (PSO)-based identification technique is applied to estimate the parameters of fuel cells in terms of the voltage-current characteristics. In [6] an effective informed adaptive PSO (EIA-PSO) algorithm with better equilibrium characteristic between global and local search is proposed. An innovative global Harmony Search algorithm for parameter estimation of PEMFC models has been introduced in [7], and compared to other state-of-the-art optimization algorithms, showing better and more robust results.

In [8], a reduced third-order model for the air supply subsystem of a PEM fuel cell is presented and experimentally validated. In [9], a model for simulation and control purposes is presented, which uses both electrical-circuit components and functional blocks to reproduce both static and dynamic behaviors of the oxygen excess ratio. In [10], a simplified mathematical model for calculating the oxygen excess ratio of a PEM fuel cell is obtained by using the mutual information (MI) approach. In [11], a first principles model is derived and validated against experimental data. The model is then linearized and used for real-time control of the air flow in a model predictive control scheme. A different approach is considered in [12], where a gain-scheduled scheme for the control of the oxygen stoichiometry in a PEM fuel cell system is implemented using a piecewise affine Linear Parameter Varying (LPV) model of the system.

Most of these modeling approaches need a prior knowledge of the model structure (usually, a first principles model) whose parameters need to be estimated. By contrast, system identification techniques allow to build mathematical models of a dynamic system based on measured data using parametric models with generic structures. In [13], a dynamic fuel cell stack model based on system identification is obtained by using a so-called Uryson-Model. A different identification approach is considered in [14], where a simple PEM fuel cell 
black-box model is proposed, which is capable of reproducing both the static and dynamic behaviors of the fuel cell. The model comprises a reduced number of passive components, and the identification technique is based on measurements of the transient response of the fuel cell.

The idea in this paper is to derive accurate PEMFC models from experimental data, suitable for model-based control approaches. Since PEMFC have a highly nonlinear dynamic behavior, it is more reasonable to estimate nonlinear models, which are able to describe the global behavior of the system over the whole operating range, rather than linear ones that are only able to approximate the system around a given operating point.

One of the most frequently studied classes of nonlinear models are the so-called block oriented models, which consist of the interconnection of Linear Time Invariant (LTI) systems and static (memoryless) nonlinearities. Within this class, Wiener models, which consist of the cascade connection of an LTI block followed by a static nonlinearity, and Hammerstein models, where the order of the linear and nonlinear blocks in the cascade connection is reversed, have been successfully used to represent dynamical systems in different application areas such as chemical processes [15], biological processes, signal processing, and control. Several methods have been proposed in the literature for the identification of Wiener models from input-output data. See for instance [15], for techniques based on over-parametrization and least squares estimation, and [16], for subspace methods.

In recent years, Support Vector Machines (SVM) regression techniques [17] have been proposed for the identification of nonlinear models represented by expansions in terms of nonlinear mappings of the model inputs. The estimates are obtained as the solution of a (convex) Quadratic Programming (QP) problem (see the recent tutorial paper [18]). Several techniques based on Least Squares SVM have been proposed for the identification of Hammerstein models [19], and WienerHammerstein models [20]. In particular, in the area of PEMFC modeling, a Least Squares SVM estimation technique based on [15], is presented in [21], and applied to identify a Hammerstein model of a PEMFC.

In this work, a new method, based on SVM regression and orthonormal bases, for the identification of Multi-Input Single-Output (MISO) Wiener models is presented and then applied to obtain a simplified model for the air supply system in a PEMFC, from experimental data. The obtained nonlinear model is thought to be used as internal model to predict the future process response in a Model Predictive Control (MPC) strategy, which has many advantages for PEMFC control [22].

The rest of the paper is organized as follows. In Section II, a parameterized model of the Wiener system is derived using Rational Orthonormal Bases. The SVM-based identification method is presented in Section III. The description of the PEMFC system is presented in Section IV. In Section V, the proposed identification method is applied to estimate a Wiener model of the air supply system in a PEMFC from measured data, and its performance is analyzed by quantifying its predictive capabilities on validation measured data. Finally, some concluding remarks are included in Section VI.

\section{Problem Formulation}

In this paper, a Multi-Input Single-Output (MISO) Wiener model is considered. The model is schematically depicted in Fig. 1 , where $\mathbf{u} \in \mathbb{R}^{m}$ is the vector input signal, $y \in \mathbb{R}$ is the scalar measured output signal, $w \in \mathbb{R}$ is additive output noise, $\mathbf{v} \in \mathbb{R}^{m}$ is the intermediate variable (output of the LTI block), $\tilde{y}$ is the output of the nonlinear block, $\mathcal{N}(\cdot)$ is the nonlinear mapping representing the static nonlinearity, and $\mathbf{G}\left(q^{-1}\right)$ is the $(m \times m)$ transfer function matrix of the LTI block.

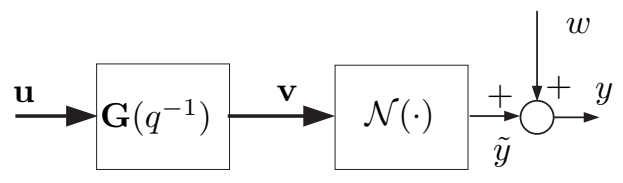

Fig. 1. Wiener Model.

The model can be described as follows:

$$
\begin{aligned}
\mathbf{v}(n) & =\mathbf{G}\left(q^{-1}\right) \mathbf{u}(n), \\
\tilde{y}(n) & =\mathcal{N}(\mathbf{v}(n)), \\
y(n) & =\tilde{y}(n)+w(n) .
\end{aligned}
$$

It is assumed that the LTI block is represented using rational orthonormal bases as follows

$$
\mathbf{G}\left(q^{-1}\right)=\sum_{\ell=1}^{p} \mathbf{b}_{\ell} \mathcal{B}_{\ell}\left(q^{-1}\right),
$$

where $\mathbf{b}_{\ell} \in \mathbb{R}^{m \times m}$ are unknown (matrix) parameters, and $\left\{\mathcal{B}_{\ell}\left(q^{-1}\right)\right\}_{\ell=1}^{\infty}$ are rational orthonormal bases ${ }^{1}$ on $H_{2}(\mathbb{T})$, the space of square integrable functions on the unit circle $\mathbb{T}$, which are analytic outside the unit disk.

In this paper, the rational Orthonormal Bases with Fixed Poles (OBFP) studied in [23], are considered. The bases are defined as

$$
\begin{aligned}
& \mathcal{B}_{\ell}(q)=\left(\frac{\sqrt{1-\left|\xi_{\ell}\right|^{2}}}{q-\xi_{\ell}}\right) \prod_{i=1}^{\ell-1}\left(\frac{1-\overline{\xi_{i}} q}{q-\xi_{i}}\right), \quad \ell \geq 2 \\
& \mathcal{B}_{1}(q)=\frac{\sqrt{1-\left|\xi_{1}\right|^{2}}}{q-\xi_{1}}
\end{aligned}
$$

and they allow prior knowledge about an arbitrary number of system modes to be incorporated in the identification process.

With this parametrization for the LTI block, the Wiener model can be represented as in Fig. 2, for the case of having $m=2$ inputs.

Defining now

$$
\begin{aligned}
& \mathbf{x} \triangleq\left[x_{1}^{1}, x_{1}^{2}, \cdots, x_{1}^{m}, \cdots, x_{p}^{1}, x_{p}^{2}, \cdots, x_{p}^{m}\right]^{T}, \\
& \mathbf{b} \triangleq\left[\mathbf{b}_{1}, \mathbf{b}_{2}, \cdots, \mathbf{b}_{p}\right], \\
& \mathbf{v} \triangleq\left[v_{1}, v_{2}, \cdots, v_{m}\right]^{T},
\end{aligned}
$$

${ }^{1}$ The bases are orthonormal in the sense that $\left\langle\mathcal{B}_{\ell}, \mathcal{B}_{k}\right\rangle=\delta_{\ell k}$, where $\delta_{\ell k}$ is the Kronecker delta, and $\langle\cdot, \cdot\rangle$ is the standard inner product in $L_{2}(\mathbb{T})$. 


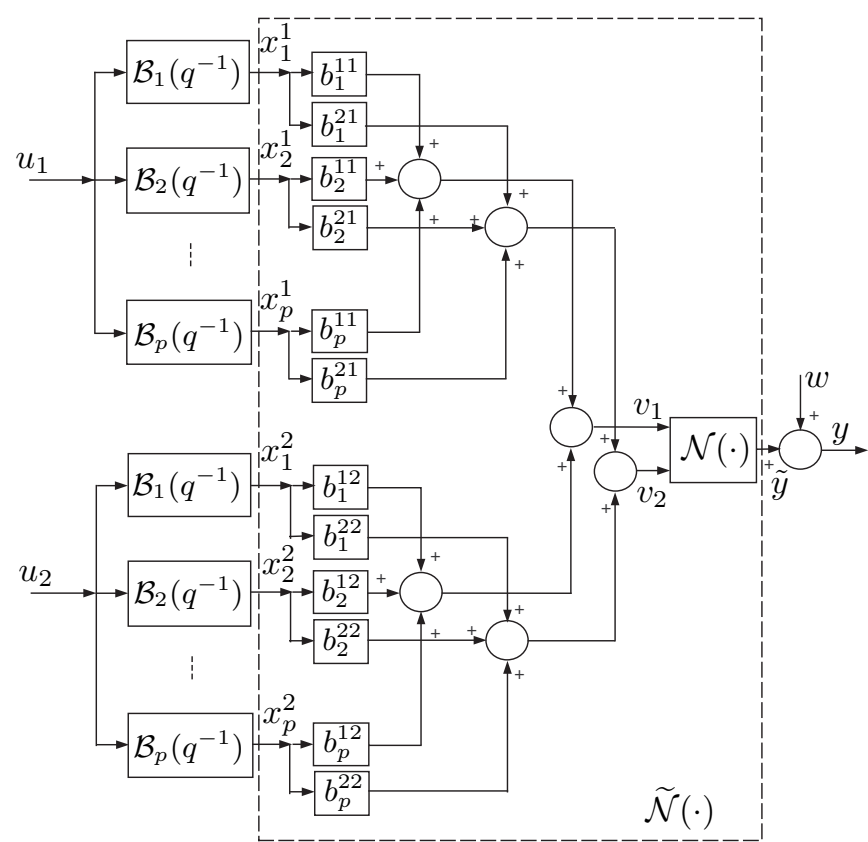

Fig. 2. Parameterized Wiener Model.

then $\mathbf{v}=\mathbf{b x}$, and eq. (2) can be written as

$$
\tilde{y}(n)=\mathcal{N}(\mathbf{v}(n))=\mathcal{N}(\mathbf{b x}(n))=\tilde{\mathcal{N}}(\mathbf{x}(n)) .
$$

Note the reader that the internal variable $\mathbf{x}(n)$ can be computed by filtering the inputs with the (known) rational orthonormal bases $\left\{\mathcal{B}_{\ell}\left(q^{-1}\right)\right\}_{\ell=1}^{p}$ used to represent the linear block in the Wiener model.

The nonlinear block $\widetilde{\mathcal{N}}(\mathbf{x}(n))$ in Fig. 2 can also be parameterized using basis functions in the form

$$
\tilde{\mathcal{N}}(\mathbf{x}(n))=\sum_{i=1}^{r} a_{i} g_{i}(\mathbf{x}(n)),
$$

where $a_{i} \in \mathbb{R}, i=1,2, \cdots, r$, are unknown parameters, and $g_{i}(\cdot): \mathbb{R}^{m p} \mapsto \mathbb{R}, i=1,2, \cdots, r$, are nonlinear basis functions.

With these parameterizations for the linear and the nonlinear blocks in the Wiener model, the output equation (3) can be written as

$$
y(n)=\mathbf{a}^{T} \mathbf{g}(\mathbf{x}(n))+w(n),
$$

where the following definitions have been done

$$
\begin{aligned}
\mathbf{a} & \triangleq\left[a_{1}, a_{2}, \cdots, a_{r}\right]^{T}, \\
\mathbf{g}(\mathbf{x}(n)) & \triangleq\left[g_{1}(\mathbf{x}(n)), g_{2}(\mathbf{x}(n)), \cdots, g_{r}(\mathbf{x}(n))\right]^{T} .
\end{aligned}
$$

In the next section, SVM methods will be used to identify the static nonlinear mapping $\widetilde{\mathcal{N}}(\cdot)$ in terms of the nonlinear basis functions $\mathbf{g}(\mathbf{x}(n))$. Note the reader that the nonlinear functions $\mathbf{g}(\mathbf{x}(n))$ need not be explicitly known, but instead they can be implicitly defined in terms of an associated kernel function (the so-called kernel trick in the machine learning literature), [24], [17].

\section{SVM-BASED IDENTIFICATION}

Equation (10) is the starting point for the formulation of the estimation problem within the framework of Support Vector regression [20]. The estimation problem, in the so-called primal weight space, can be formulated as follows: Given a data set of measured inputs and outputs $\{u(n), y(n)\}_{n=1}^{N}$, the goal is to estimate a model of the form

$$
y(n)=\mathbf{a}^{T} \tilde{\mathbf{g}}(\mathbf{x}(n))+c+\nu(n),
$$

where $c$ is a bias term, and $\{\nu(n)\}$ is an i.i.d. random process with zero mean and finite variance. The unknowns in the model are $\mathbf{a} \in \mathbb{R}^{r}, c \in \mathbb{R}$, and the order $r$.

It is well known that the unknowns a and $c$ can be determined by solving the following constrained optimization problem [17]

$$
\min _{\mathbf{a}, c, \nu} \quad \frac{1}{2} \mathbf{a}^{T} \mathbf{a}+\gamma \sum_{n=1}^{N} L_{\epsilon}(\nu(n))
$$

subject to

$$
\begin{aligned}
& y(n)-\mathbf{a}^{T} \tilde{\mathbf{g}}(\mathbf{x}(n))-c-\nu(n)=0, \\
& n=1, \cdots, N
\end{aligned}
$$

where $\gamma>0$ is a regularization constant providing a tradeoff between model complexity (penalized by the first term in (12)) and fitting accuracy to the experimental data (penalized by the second term in (12)), and $L_{\epsilon}(\nu(n))$ is Vapnik's $\epsilon$-insensitivity loss function, defined as

$$
L_{\epsilon}(\nu(n))= \begin{cases}|\nu(n)|-\epsilon & \text { if }|\nu(n)| \geq \epsilon \\ 0 & \text { otherwise }\end{cases}
$$

The optimization problem (12) can be solved more easily in its dual formulation using Lagrange multipliers, [25]. Introducing the positive definite kernels [17]

$$
K(\mathbf{x}(n), \mathbf{x}(k)) \triangleq \tilde{\mathbf{g}}^{T}(\mathbf{x}(n)) \tilde{\mathbf{g}}(\mathbf{x}(k))
$$

associated with the functions $\tilde{\mathbf{g}}(\mathbf{x}(n))$, the dual problem in the Lagrange multipliers $\left(\alpha_{n}\right.$ and $\left.\alpha_{n}^{*}\right)$ can be formulated as follows:

$$
\begin{aligned}
\max _{\alpha_{n}, \alpha_{n}^{*}} & -\frac{1}{2} \sum_{n, k=1}^{N}\left(\alpha_{n}-\alpha_{n}^{*}\right)\left(\alpha_{k}-\alpha_{k}^{*}\right) K(\mathbf{x}(n), \mathbf{x}(k)) \\
& -\epsilon \sum_{n=1}^{N}\left(\alpha_{n}+\alpha_{n}^{*}\right)+\sum_{n=1}^{N} y(n)\left(\alpha_{n}-\alpha_{n}^{*}\right) \\
\text { subject to } & \sum_{n=1}^{N}\left(\alpha_{n}-\alpha_{n}^{*}\right)=0 \\
& \alpha_{n}, \alpha_{n}^{*} \in[0, \gamma], \quad n=1, \cdots, N
\end{aligned}
$$

which is a quadratic programming (QP) problem with box constraints, [18]. The dual model representation is then given by

$$
y(n)=\sum_{k=1}^{N}\left(\alpha_{k}-\alpha_{k}^{*}\right) K(\mathbf{x}(n), \mathbf{x}(k))+c
$$

Although the number of terms in the representation (15) equals the number of data points $N$, only a reduced number 
of terms, corresponding to a small number of vectors $\mathbf{x}(k)$, will have non vanishing coefficients $\left(\alpha_{k}-\alpha_{k}^{*}\right)$. These vectors are the so-called support vectors. The number of support vectors will depend on the chosen values for $\epsilon$ and $\gamma$, and on the chosen kernel function. Commonly used kernels are Gaussian Radial Basis Functions (RBF), polynomial kernels, and MultiLayer Perceptrons (MLP).

\section{PEM Fuel Cell System}

The air supply in PEMFC results critical in the system performance because the oxygen reacts instantly as current is drawn, whereas the oxygen supply is limited by the dynamics of the inlet manifold and the air compressor. The time constants of the electrochemical reactions are in the order of magnitude of $10^{-19} \mathrm{~s}$. Thus, for control purposes, these time constants can be assumed as negligible in comparison to the ones associated with the other (much slower) processes involved, such as temperature evolution $\left(10^{2} s\right)$, and dynamics of volume filling $\left(10^{-1} \mathrm{~s}\right)$.

The air flow excess is reflected by the oxygen excess ratio, which is the ratio of oxygen supplied to oxygen used in the cathode. Control of the oxygen excess ratio is usually approached through the manipulation of the compressor motor voltage, while the current being drawn can be considered as a disturbance [3], [26], [27].

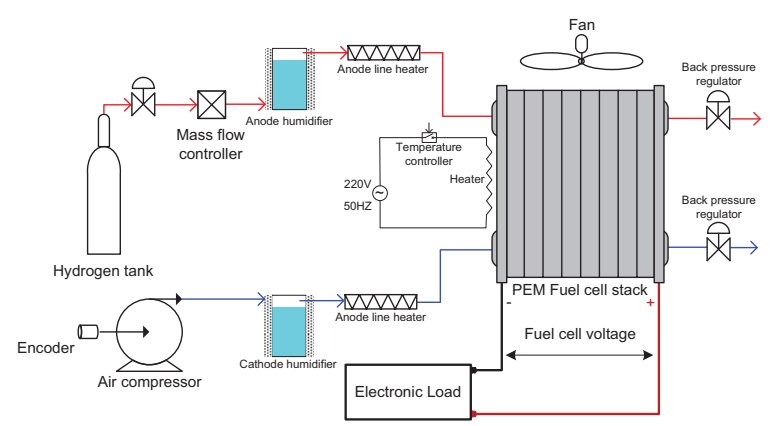

Fig. 3. Simplified schematic diagram of the fuel cell test station.

A simplified schematic diagram of the laboratory PEMFC used in this paper is depicted in Fig. 3, where the main components of the fuel cell test station can be observed. The principal elements are a fuel cell stack, an air compressor, hydrogen storage tanks, gases manifolds, humidifiers, line heaters and valves. The fuel cell stack is a ZBT ${ }^{\mathrm{R}}$ 8-cell stack with Nafion $115^{\mathrm{R}}$ membrane electrode assemblies (MEAs), $50 \mathrm{~cm}^{2}$ of active area and $150 \mathrm{~W}$ power. The membrane exchange humidifiers used to maintain proper humidity conditions inside the cells are Cellkraft ${ }^{\circledR}$. The air compressor consists in a $12 V$ DC oil-free diaphragm vacuum pump. The line heaters and stack temperatures are controlled using decentralized PID controllers, allowing independent gas conditions (humidity and temperature) inside the stack [28]. Fig. 4 shows a picture of the fuel cell test station studied in this paper.

The data acquisition and control system is composed by a Host computer and a RTOS (Real Time Operating System)

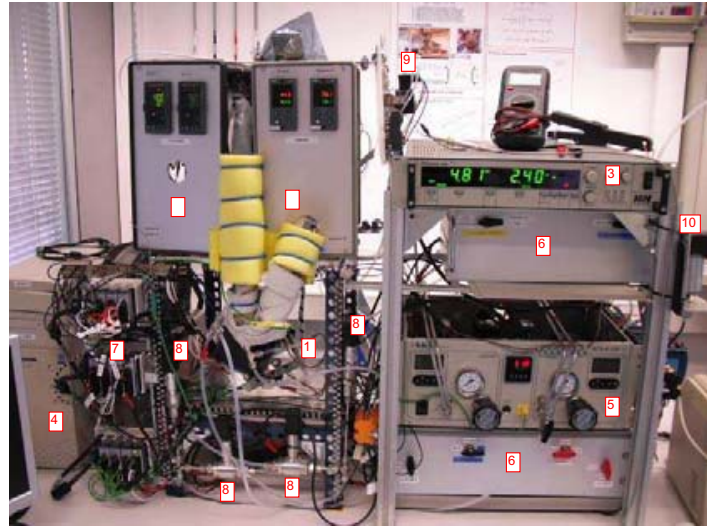

Fig. 4. Fuel cell test station. 1: Fuel cell stack; 2: humidifiers; 3: electronic load; 4: real-time computer; 5: back-pressure regulators; 6: valves; 7: acquisition and control cartridges; 8: pressure sensors; 9: cathode outlet proportional valve; 10: air compressor.

computer running in real time. The RTOS computer communicates with the input/output $(I / O)$ modules, made by National Instrument ${ }^{\circledR}$, throughout an FPGA (Field Programmable Gate Array) target and a PCI bus. The two computers are connected via ethernet. The Host computer also allows monitoring the evolution of the variables and commanding the system through a graphical interface developed in LabVIEW ${ }^{\circledR}$. An extensive amount of variables are measured and recorded every $100 \mathrm{~ms}$.

\section{IDENTIFICATION RESULTS}

The SVM-based identification method introduced in Section III is used here to estimate a Wiener model for the air supply system of the PEMFC described in Section IV. The compressor motor voltage and the current being drawn from the PEMFC (denoted hereafter as $u_{1}$ and $u_{2}$, respectively), are considered as the inputs to the system, while the oxygen excess ratio (denoted hereafter as $y$ ) is considered as the output.

For the purposes of identification, multilevel random signals were applied to the inputs $u_{1}$ and $u_{2}$, and 2500 samples of the inputs and the output were collected with a sampling period $T_{s}=0.1 \mathrm{~s}$. The input-output data are shown in Fig. 5. The first 2000 samples were used for the estimation, while the remaining 500 samples were used for validation purposes.

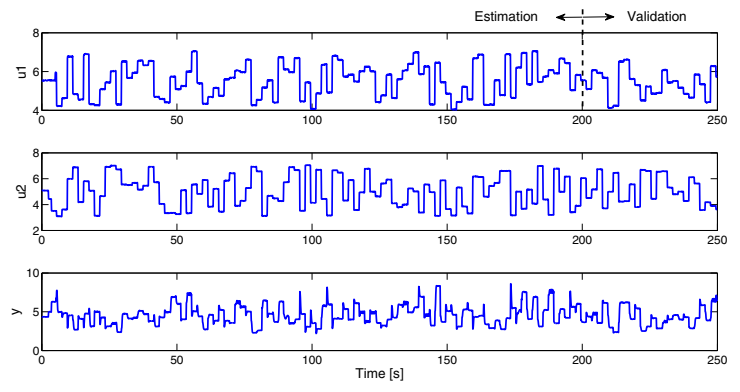

Fig. 5. Input-Output Estimation and Validation Measured Data.

For the identification experiments, the design parameters of the SVM method were set to $\epsilon=0.05, \gamma=2000$, and $\sigma^{2}=2$. 


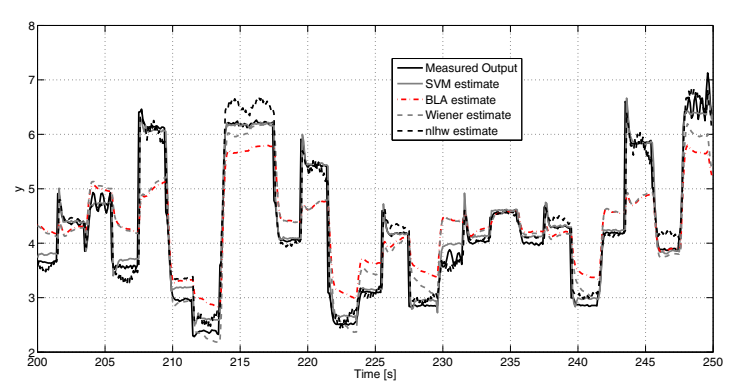

Fig. 6. Measured Output (black solid line), SVM-based estimated output (grey solid line), BLA-based estimated output (red dash-dotted line), Wiener model estimated output (grey dashed line), and nlhw-based estimated output (black dashed line).

The rational orthonormal bases with fixed poles described in Section II are used to represent the linear block in the Wiener model, with poles located at $\xi=0.1$ (i.e., Laguerre bases), and model order equal to 4 .

The measured output (black solid line) and the output predicted by the SVM-based model (red dashed line) are shown in Fig. 6, where a good agreement between them can be observed. The Best Fit $^{2}$ between the measured and the predicted outputs is $74.8970 \%$. The number of support vectors resulted 1545 .

The same estimation/validation data are used to estimate the Best Linear Approximation (BLA) of the system resorting to the CVA subspace algorithm as implemented in the n4sid function of the System Identification Toolbox for use with Matlab. The function computes the best model order, which resulted $n=3$. The output predicted by the BLA model, for the validation input, is depicted in red dash-dotted line in Fig. 6 . The Best Fit between the measured and the predicted output is $48.4976 \%$. This result clearly indicates that a linear model is not capable to accurately represent the PEMFC when large deviations from the operating point are considered.

For the purposes of comparison, the same estimation/validation data are used to estimate a Wiener model as in Fig.1, where the 2-Inputs 1-Output, 3rd order, BLA model is assigned to the estimated linear block, and the nonlinear SISO block is given a representation of the form:

$$
\mathcal{N}(v(n))=\sum_{i=1}^{r} a_{i} g_{i}(v(n)),
$$

where now, polynomials are considered for the scalar nonlinear functions $g_{i}(v(n))$. The unknown coefficients $a_{i}$ are then computed by least squares fitting to the estimation data. A 9-th order odd polynomial was estimated. The output predicted by this Wiener model is depicted in grey dashed line in Fig. 6. The Best Fit between the measured and the predicted output is $54.7914 \%$. It can be observed that there are improvements

\footnotetext{
${ }^{2}$ The Best FIT is defined as BestFIT $=\left(1-\frac{\left\|Y-Y_{v}\right\|}{\left\|Y_{v}-y_{m e a n}\right\|}\right) \times 100$, where $Y$ is a vector with the output of the model when excited with the validation input data, $Y_{v}$ is a vector with the validation output data, and $y_{\text {mean }}$ is the mean value of the validation output.
}

in the prediction accuracy with respect to the BLA model, but this model is not able to outperform the prediction accuracy of the SVM-based estimated model. Also for the purposes of comparison, the same data are used to estimate a Wiener model resorting to the nlhw function in the System Identification Toolbox. The estimated Wiener model consists of a 7 th order linear block in cascade with a 9-th order polynomial nonlinear block. The output predicted by this Wiener model is depicted in black dashed line in Fig. 6. The Best Fit between the measured and the predicted output is $69.6265 \%$. As it can be observed, there are improvements in the estimation accuracy but still this model is not able to outperform the prediction accuracy of the SVM-based estimated model. Table I, summarizes the results of the estimation accuracy for the four identification techniques considered in this section.

TABLE I

ESTIMATION ACCURACY FOR THE FOUR IDENTIFICATION TECHNIQUES.

\begin{tabular}{rcccc}
\hline Model & SVM & Wiener & BLA & nlhw \\
\hline BestFIT [\%] & $\mathbf{7 4 . 8 9 7 0}$ & 54.7914 & 48.4976 & 69.6265 \\
\hline
\end{tabular}

To investigate how does the size of the $\epsilon$-tube affect the estimation accuracy and the resulting model complexity (number of support vectors), identification experiments with different values of $\epsilon$ in the range $[0.005,0.3]$, were carried out. The results are shown in Fig. 7, where the Best FIT as a function of $\epsilon$ (top plot), and the number of Support Vectors as a function of $\epsilon$ (bottom plot) are displayed. As expected, both the estimation accuracy and the model complexity decrease as $\epsilon$ increases. For these experiments, the remaining parameters of the SVMbased identification method were set to $\gamma=2000$ and $\sigma^{2}=2$
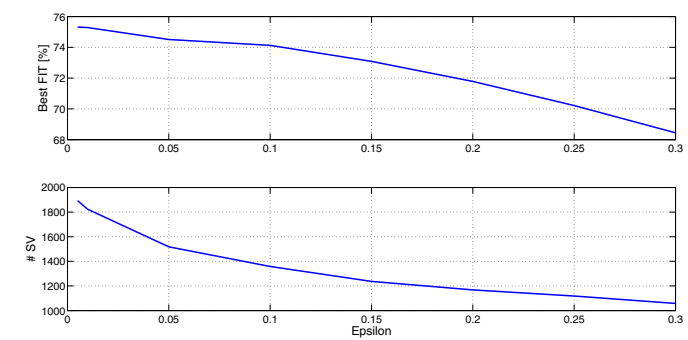

Fig. 7. Top plot: Best FIT vs. $\epsilon$. Bottom plot: Number of SV vs. $\epsilon$

To evaluate the influence that the location of the poles of the basis functions has on the prediction accuracy of the estimated model and the resulting model complexity, identification experiments with different values of the Laguerre parameter (i.e., the pole location $\xi$ of the Laguerre bases) within the range $[0.05,0.75]$ were carried out. The results are shown in Fig. 8 where the Best FIT as a function of the Laguerre parameter (top plot) and the number of support vectors as a function of the Laguerre parameter (bottom plot) are displayed. As it can be observed from the top plot, the optimal value of the Laguerre parameter, regarding estimation accuracy, is 
0.05, and the accuracy decreases as the Laguerre parameter increases. On the other hand, as the Laguerre parameter increases above the optimal value, the resulting number of support vectors decreases (see bottom plot).
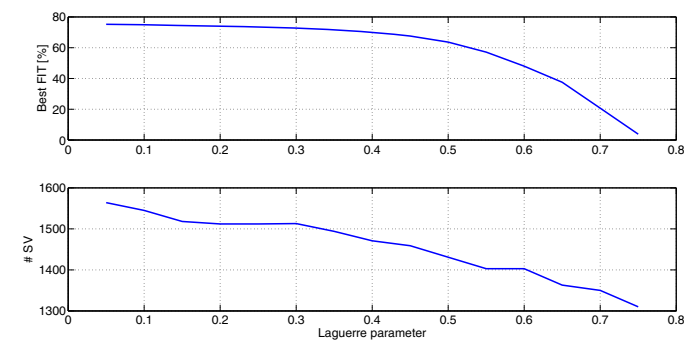

Fig. 8. Top plot: Best FIT vs. Laguerre parameter $\xi$. Bottom plot: Number of SV vs. Laguerre parameter $\xi$.

\section{CONCLUSIONS}

In this paper, a new MISO Wiener model identification method based on Support Vector Regression and orthonormal bases is introduced and applied to estimate a simplified model for the air supply system of a PEMFC from measured data. It is shown that the estimated model has good predictive capabilities even when large deviations from the PEMFC operating point are considered. This is not the case for the BLA of the system, which results with poor predictive capabilities.

\section{ACKNOWLEDGEMENTS}

The authors would like to thank the proficient technical staff of the Fuel Cells Laboratory at the Institut of Robótica i Informática Industrial (CSIC-UPC, Barcelona), where all the experimental tests were performed.

\section{REFERENCES}

[1] J. Larminie and A. Dicks, Fuel Cell Systems Explained, 2nd ed. Wiley and Sons, 2003.

[2] J. Pukrushpan, A. Stefanopoulou, and H. Peng, "Control of fuel cell breathing," IEEE Control Systems Magazine, vol. 24, no. 2, pp. 30-46, 2004.

[3] J. Pukrushpan, H. Peng, and A. Stefanopoulou, "Control-oriented modelling and analysis for automotive fuel cell systems," Journal of Dynamic Systems, Measurement and Control, vol. 126, pp. 14-25, 2004.

[4] Z.-J. Mo, X.-J. Zhu, L.-Y. Wei, and G.-Y. Cao, "Parameter optimization for a PEMFC model with a hybrid genetic algorithm," International Journal of Energy Research, vol. 30, no. 8, pp. 585-597, 2006.

[5] M. Ye, X. Wang, and Y. Xu, "Parameter identification for proton exchange membrane fuel cell model using particle swarm optimization," International Journal of Hydrogen Energy, vol. 34, no. 2, pp. 981-989, 2009.

[6] Q. Li, W. Chen, Y. Wang, S. Liu, and J. Jia, "Parameter identification for PEM fuel-cell mechanism model based on effective informed adaptive particle swarm optimization," Industrial Electronics, IEEE Transactions on, vol. 58, no. 6, pp. 2410-2419, 2011.

[7] A. Askarzadeh and A. Rezazadeh, "An innovative global harmony search algorithm for parameter identification of a PEM fuel cell model," IEEE Transactions on Industrial Electronics, vol. 59, no. 9, pp. 3473-3480, 2012.

[8] R. Talj, D. Hissel, R. Ortega, M. Becherif, and M. Hilairet, "Experimental validation of a PEM fuel-cell reduced-order model and a motocompressor higher order sliding-mode control," Industrial Electronics, IEEE Transactions on, vol. 57, no. 6, pp. 1906-1913, June 2010.
[9] C. Ramos-Paja, R. Giral, L. Martinez-Salamero, J. Romano, A. Romero, and G. Spagnuolo, "A PEM fuel-cell model featuring oxygen-excessratio estimation and power-electronics interaction," Industrial Electronics, IEEE Transactions on, vol. 57, no. 6, pp. 1914-1924, June 2010.

[10] C. Restrepo, T. Konjedic, C. Guarnizo, O. Avino-Salvado, J. Calvente, A. Romero, and R. Giral, "Simplified mathematical model for calculating the oxygen excess ratio of a PEM fuel cell system in real-time applications," Industrial Electronics, IEEE Transactions on, vol. 61, no. 6, pp. 2816-2825, June 2014.

[11] Y.-X. Wang and Y.-B. Kim, "Real-time control for air excess ratio of a PEM fuel cell system," Mechatronics, IEEE/ASME Transactions on, vol. 19 , no. 3, pp. 852-861, June 2014.

[12] F. Bianchi, C. Kunusch, C. Ocampo-Martinez, and R. Sanchez-Pena, "A gain-scheduled LPV control for oxygen stoichiometry regulation in PEM fuel cell systems," Control Systems Technology, IEEE Transactions on, vol. 22, no. 5, pp. 1837-1844, Sept 2014.

[13] M. Meiler, O. Schmid, M. Schudy, and E. P. Hofer, "Dynamic fuel cell stack model for real-time simulation based on system identification," Journal of Power Sources, vol. 176, no. 2, pp. 523-528, 2008

[14] C. Raga, A. Barrado, A. Lázaro, C. Fernández, V. Valdivia, I. Quesada, and L. Gauchía, "Black-box model, identification technique and frequency analysis for PEM fuel cell with overshooted transient response," IEEE Transactions on Power Electronics, vol. 29, no. 10, pp. 5334-5346, October 2014

[15] J. Gómez and E. Baeyens, "Identification of block-oriented nonlinear systems using orthonormal bases," Journal of Process Control, vol. 14, no. 6, pp. 685-697, 2004.

[16] - "Subspace identification of multivariable Hammerstein and Wiener models," European Journal of Control, vol. 11, no. 2, pp. 127136, 2005.

[17] V. Vapnik, Statistical Learning Theory. New York: John Wiley \& Sons, 1998.

[18] J. Suykens, "Support vector machines and kernel-based learning for dynamical systems modelling," in Proc. of the 15th IFAC SYSID, SaintMalo, France, July 2009, pp. 1029-1037.

[19] I. Goethals, K. Pelckmans, J. Suykens, and B. De Moor, "Identification of MIMO Hammerstein models using least squares support vector machines," Automatica, vol. 41, pp. 1263-1272, 2005.

[20] T. Falck, P. Dreesen, K. De Brabanter, K. Pelckmans, B. De Moor, and J. Suykens, "Least-squares support vector machines for the identification of Wiener-Hammerstein systems," Control Engineering Practice, vol. 20, no. 11, pp. 1165-1174, 2012.

[21] C.-H. Li, X.-J. Zhu, G.-Y. Cao, S. Sui, and M.-R. Hu, "Identification of the Hammerstein model of a PEMFC stack based on least squares support vector machines," Journal of Power Sources, vol. 175, pp. 303316, 2008.

[22] D. Feroldi, M. Serra, and J. Riera, "Performance improvement of a PEMFC system controlling the cathode outlet air flow," J. of Power Sources, vol. 169, no. 1, pp. 205-212, 2007.

[23] B. Ninness and F. Gustafsson, "A unifying construction of orthonormal bases for system identification," IEEE Trans. on Autom. Control, vol. AC-42, no. 4, pp. 515-521, 1997.

[24] V. Vapnik, The Nature of Statistical Learning Theory. New York: Springer-Verlag, 1995

[25] B. Schölkopf and A. Smola, Learning with Kernels. Cambridge, MA: MIT Press, 2002.

[26] M. Grujicic, K. Chittajallu, E. Law, and J. Pukrushpan, "Model-based control strategies in the dynamic interaction of air supply and fuel cell," Proceedings of the Institution of Mechanical Engineers, Part A: J. of Power and Energy, vol. 218, no. 7, pp. 487-499, 2004.

[27] J. Golbert and D. Lewin, "Model-based control of fuel cells: (1) regulatory control," J. of Power Sources, vol. 135, pp. 135-151, 2004.

[28] C. K. A. Niknezhadi, M. Allué-Fantova and C. Ocampo-Martínez, "Design and implementation of LQR/LQG strategies for oxygen stoichiometry control in PEM fuel cells based systems," Journal of Power Sources, vol. 196, pp. 4277-4282, 2010. 